\title{
Explorer les compétences rédactionnelles au lycée professionnel
}

\author{
Marie-Paule Jacques ${ }^{1,2}$ et Cindy De Amaral $^{2}$ \\ ${ }^{1}$ ESPE, Université Grenoble Alpes \\ ${ }^{2}$ LIDILEM, Université Grenoble Alpes, Bâtiment Stendhal, CS40700, 38058 Grenoble Cedex 9, France
}

\begin{abstract}
Résumé. Nous proposons dans cet article une analyse de productions textuelles d'élèves de Seconde de la filière de baccalauréat GestionAdministration. Notre hypothèse est que ces élèves de lycée professionnel mettent en œuvre dans leurs productions écrites un certain nombre d'habiletés rhétoriques et communicationnelles qui, masquées par les aspects formels de maitrise de la langue, pourraient constituer une base solide à l'enseignement des compétences rédactionnelles. Nous nous inscrivons dans le champ de recherches autour des "compétences ignorées » des élèves, qui considère les habiletés construites dans les pratiques extrascolaires comme devant être repérées et reconnues. Nous mettons en évidence, par l'analyse de courriels répondant à un scénario pédagogique proche de l'univers professionnel auquel ces élèves se destinent, de réelles compétences de communication, voisinant des habiletés rhétoriques et énonciatives contrastées, et de réelles défaillances sur les plans formel et pragmatique. Nous en déduisons une proposition didactique dont nous exposons les grandes lignes, et qui doit être mise à l'épreuve auprès de ces mêmes élèves.
\end{abstract}

\begin{abstract}
Exploring writing skills in vocational education context. In this article, we analyze emails produced by pupils in the context of vocational education and training, the French «baccalauréat Gestion-Administration ». We hypothesize that these pupils master several writing skills, in particular in communication and in rhetorics, while they fail in other areas, as spelling. Their poor spelling, as the visible part of their writing, hides their ability to successfully manage communication and gives them a sense of failure. However, from a day-to-day practice of Internet communication, these teenagers have developed unknown skills that they do not transfer to education. Our purpose is to highlight these abilities, displayed by the analysis of emails produced in a classroom task, which is related to the vocational training they receive. Our analysis shows that they success in assessing the situation, peculiarly the hierarchical relation to the addressee, while they usually fail to produce a text totally compliant with the norms of the written language. We draw from this teaching suggestions that are intended to be experimented with these pupils.
\end{abstract}

1 Corresponding author : marie-paule.jacques@univ-grenoble-alpes.fr 


\section{Introduction}

Écrire est une conquête qui ouvre le champ de la formation personnelle et professionnelle et qui dote de compétences valorisées socialement et professionnellement. L'irruption dans la vie sociale d'écrans aux fonctions multiples et de moyens de communications divers et variés n'a pas relégué l'écriture au rang de technologie dépassée, elle contribue au contraire à diversifier les fonctions de l'écrit et à agrandir la palette des compétences à construire pour les maitriser [1]. L'école joue un rôle majeur pour le développement de ces compétences. Tout au long de la scolarité obligatoire, les élèves sont exposés à de multiples situations de production d'écrits, censées leur permettre de construire des habiletés rédactionnelles variées.

Nous proposons dans cet article une analyse de productions d'élèves de Seconde de baccalauréat professionnel Gestion-Administration (15-17 ans), dans l'objectif de mettre en évidence et valoriser les compétences rédactionnelles de ces élèves et de dégager des pistes didactiques susceptibles d'agir positivement sur leur rapport à l'écrit, qui constitue un levier didactique potentiel [2-3].

En fonction des conceptions de ce que signifie « écrire », de la conscience des élèves de leur propre maitrise de l'écrit, des transferts qu'ils sont à même d'opérer entre pratiques scolaires et pratiques extrascolaires de lecture et d'écriture, se construit un rapport à l'écrit et à l'écriture qui imprègne durablement leur rapport à l'école [3], et qui peut constituer un obstacle ou un facilitateur. Parmi les représentations courantes, sont tenaces la notion de l'écriture comme don ou encore l'idée que l'écriture procède uniquement de la transcription sur le papier (ou maintenant via le traitement de textes) d'un matériau déjà achevé, et ne comporte donc ni brouillon, ni phase de réécriture, révision, essais et modifications [4]. Ainsi, lorsque la production effective ne satisfait pas les normes scolaires, le scripteur est-il enclin à se disqualifier comme sujet scripteur, en raison du fait que "L'écriture impose des modèles... syntaxiques, textuels qui pèsent sur le scripteur en raison inverse de sa compétence à les maitriser. » [Dabène, 1990 : 17, cité par 4]. Une enquête en cours auprès d'une quarantaine d'élèves de seconde (dans le cadre du travail doctoral de C. De Amaral) montre que ces élèves assimilent volontiers la qualité de la production écrite à la correction formelle: "écrire bien» c'est "écrire sans fautes». A contrario, une piètre qualité formelle de l'écrit ne pourrait permettre «d'écrire bien». On peut alors présumer que certains de ces élèves se trouvent dans une situation de relative insécurité scripturale qui fait obstacle à la reconnaissance et au développement de leurs compétences rédactionnelles.

En même temps, les filières professionnelles, alors même qu'elles scolarisent actuellement $1 / 3$ des lycéens en France, n'ont donné lieu qu'à peu de travaux en didactique de l'écrit. Ce terrain a été exploré par la sociologie de l'éducation, qui a montré que le public de lycée professionnel est très marqué sur le plan sociologique et que la plupart des élèves sont orientés vers le lycée professionnel non par choix mais par un processus d'orientation par l'échec ou d'auto-sélection [5]. Les difficultés d'ordre socio-didactique établies par la recherche sont particulièrement présentes pour les élèves des filières du tertiaire, parmi lesquelles celle de Gestion-Administration à laquelle nous nous intéressons dans cet article. Selon un rapport récent du Cnesco [6], cette filière cristallise les difficultés puisqu'on y retrouve une forte proportion d'élèves issus de milieux populaires et / ou de l'immigration, élèves qui, selon Jellab [7] et Charlot [8], sont particulièrement démunis en termes de « capital scolaire ».

Pourtant, la littérature didactique s'est encore peu penchée sur ce public spécifique, surtout sous l'angle de l'écrit, alors que, selon Lahire [9], l'écrit et surtout, l'entrée dans un rapport écrit aux savoirs, est un enjeu majeur de réussite sociale et scolaire dans notre société. On peut donc penser que la didactique de l'écrit gagnera à explorer davantage ce terrain. 
Notre hypothèse est que ces élèves de lycée professionnel mettent en œuvre dans leurs productions écrites un certain nombre d'habiletés rhétoriques et communicationnelles qui sont masquées par les aspects formels de maitrise de la langue. Notre propos ici est de mettre en regard les compétences communicationnelles et discursives manifestées dans la production de courriels, en réponse à un exercice scolaire, et les défaillances formelles susceptibles de discréditer ces écrits, en ce qu'elles sont assimilées, aux yeux mêmes des élèves et au regard de la norme scolaire, à un défaut de maitrise de l'écrit. Nous nous inscrivons ainsi dans le champ des recherches autour des "compétences ignorées » des élèves, qui sera évoqué dans la section suivante avant un rappel des travaux consacrés aux écrits professionnels. Nous expliciterons ensuite notre méthode d'analyse des productions avant d'en indiquer les résultats et enfin de conclure sur les pistes didactiques à envisager.

\section{De l'écriture personnelle à l'écriture professionnelle}

\subsection{Les « compétences ignorées 》 des élèves}

L'institution scolaire dresse un tableau assez négatif des compétences rédactionnelles des élèves de lycée professionnel, le plus souvent en termes de déficit ou d'incapacité, discours négatif qui s'explique notamment par de réelles contre-performances sur les plans orthographiques et/ ou grammaticaux. En effet, comme l'a bien identifié Colin [10] lors de son analyse de discours d'enseignants de cycle 3 , les enseignants se focalisent souvent sur les aspects rédactionnels les plus formels, liés à la "maitrise de la langue », éléments qui font parfois défaut aux élèves de lycée professionnel. Or ces élèves, comme tous les adolescents, disposent à l'égard de l'écrit de compétences «ignorées à au moins trois titres : soit parce que les sujets ignorent qu'ils les possèdent, soit parce que l'institution n'en a pas connaissance ou encore parce que les sujets, bien qu'ils aient certaines connaissances, ne les mobilisent pas dans un cadre institutionnel où elles ne sont pas reconnues » [11]. L'absence de reconnaissance évoquée est due en partie au fait que ces compétences sont construites en dehors du temps et des supports scolaires. Elles sont cependant transférables ou transposables dans l'univers scolaire, comme le montre Elalouf [11] en soulignant que, lors de l'enquête internationale PISA en compréhension de l'écrit de 2009, où les élèves ont pour la première fois passé des épreuves à la fois sur support papier et sur support numérique, cette dualité a permis une comparaison de la quantité et de la qualité des réponses qui montre un avantage assez constant pour le support numérique.

Quoiqu'il n'y ait pas d'effet magique du support numérique, celui-ci a la particularité de susciter chez les adolescents des pratiques de lecture et d'écriture quotidiennes. Les chroniques, récits fictifs écrits par des adolescents ou de jeunes adultes et publiés sous la formes d'épisodes, et les forums notamment sont très populaires chez les adolescents. La participation à un forum, en ce qu'elle suppose la co-construction des normes du bien écrire, peut en particulier conduire à des commentaires métacommunicatifs, métadiscursifs et métalinguistiques [12], qui sont autant de temps de compréhension de ce qui concourt à la recevabilité et à l'efficacité de l'écrit - pour, par exemple, retrouver le titre d'une chanson, s'informer ou échanger des opinions. Ces pratiques dotent les élèves d'habiletés, d'un « déjà-là » sur lequel l'enseignement gagnerait à s'appuyer [13], pour en valoriser les transferts vers les activités scolaires. L'analyse que nous présenterons plus loin s'inscrit dans cette perspective de valorisation des savoirs et savoir-faire des élèves dans l'objectif de réhabiliter, y compris à leurs propres yeux, les compétences de communication dont ils font montre. Celles-ci sont en effet une composante particulièrement centrale de nombre d'écrits que les élèves des filières de Gestion-Administration doivent être capables de produire dans les univers professionnels que leur formation les destine à intégrer. 


\section{2 Écriture professionnelle, écriture numérique : le courriel}

Si l'écriture professionnelle fait l'objet d'un grand nombre de manuels pratiques [14] et d'un nombre grandissant de recherches [voir par exemple 15-17], le champ des compétences rédactionnelles dans son ensemble demeure difficile à circonscrire. En effet, les manuels s'accordent sur la nécessité toujours plus grande, toutes professions confondues, de produire des écrits efficaces sur le plan communicationnel, mais la recherche montre qu'il est difficile de définir précisément les genres de textes que des futurs professionnels auront à maitriser, ainsi que les attentes spécifiques à l'écriture professionnelle. Par exemple, sous une même dénomination compte-rendu, peuvent être rangées des productions réalisant de manières très diversifiées l'objectif de garder une trace d'une réunion formelle, ce qui tendrait à dévaluer un enseignement fondé sur les genres [18]. Les travaux ayant exploré ce terrain de l'écrit professionnel s'accordent pour dire qu'un certain nombre de compétences transversales, liées tant à la maitrise de la langue qu'aux compétences communicationnelles, sont nécessaires à l'insertion professionnelle [18-19]. C'est l'hypothèse qui sous-tend le projet CertiRedac [20] : «même si les écrits professionnels sont variés ( $c f$. Laborde-Milaa et al., dir., 2014) et que la formation doit tenir compte des genres spécifiques aux diverses situations professionnelles, il demeure néanmoins possible de trouver un dénominateur suffisamment commun aux compétences rédactionnelles mises en œuvre dans la majorité des écrits professionnels ». On peut donc supposer qu'au-delà des genres particuliers des univers professionnels ciblés par le bac professionnel Gestion-Administration, certaines des habiletés repérées à travers la production d'un texte dans un genre particulier seront exploitables dans un autre genre. On verra que notre démarche d'analyse mêle ainsi la prise en compte d'éléments spécifiques au genre et d'éléments plus transverses.

Le genre que nous avons choisi de travailler, le courrier électronique (courriel ou email), joue un rôle de plus en plus important dans le cadre de l'activité professionnelle. Ce genre est un mode d'échanges en expansion depuis les années 1990 au sein de l'entreprise [21]. Il pose à son auteur un certain nombre de problèmes par ses aspects partiellement informels qui le situent aux frontières de l'oral et de l'écrit [21], ce qui, compte tenu du profil des élèves de lycée professionnel, le rend particulièrement intéressant à observer. Le fait qu'il puisse être combiné à d'autres modes de communication (notes internes, appels téléphoniques, échanges oraux) pose également le problème du degré de «recontextualisation » dont les élèves sont capables puisque, selon Lahire [22], les élèves n'entretenant pas un rapport écrit au monde et aux savoirs éprouvent de grandes difficultés à décontextualiser et recontextualiser les échanges. Enfin, à la lumière de différents travaux [21-23], il semblerait que ce genre, bien que très présent dans la vie quotidienne, ait été peu codifié. D'après [24], il emprunte au billet son caractère moins formel que celui d'une lettre et présente en même temps des traits manifestant son inscription dans un échange de nature dialogique, comme la conversation. Toutefois, la situation de face-à-face d'une conversation ou d'un dialogue in presentia n'étant pas réalisée, les ajustements et le recours au contexte qu'elle permet ne sont pas disponibles. Ces particularités en font un genre hybride, présentant des traits mouvants, dont la réalisation doit être avant tout guidée par la saisie pragmatique du contexte dans lequel il s'inscrit. Son niveau de formalisme est dépendant tout à la fois de ses objectifs, de la nature des relations hiérarchiques entre l'émetteur et le destinataire, de sa place dans un échange - un courriel de réponse peut être plus économe en salutations que celui qui initie l'échange. Il est donc un genre privilégié pour la saisie de compétences communicationnelles.

Nos analyses portent sur un petit corpus de courriels, que nous décrivons maintenant, avec notre méthodologie.

\section{Corpus et méthode d'analyse}




\subsection{Corpus}

L'étude qui est présentée ici a pour cadre un projet de recherche-action financé par l'Institut Carnot de l'Éducation. Ce projet a pour objectifs l'exploration des compétences rédactionnelles d'élèves du baccalauréat professionnel Gestion-Administration et la formulation de propositions didactiques pour alimenter les séances d'atelier rédactionnel. L'atelier rédactionnel est un dispositif prévu par le référentiel de ce baccalauréat depuis 2014, qui a pour particularité d'instituer une pratique d'enseignement en co-animation entre enseignant de discipline professionnelle et enseignant de lettres, dans une perspective d'enseignement de l'écriture professionnelle.

Au cours de cet atelier, les enseignantes de la classe ont travaillé durant trois séances sur la production de courriers électroniques. Les écrits analysés ici correspondent aux productions collectées auprès d'élèves de Seconde lors de la dernière des trois séances, qui s'inscrivaient dans le "scénario pédagogique » suivant. Il s'agissait d'organiser un séminaire dans une usine de parfum pour une entreprise de fleuriste fictive souhaitant se réorienter vers les produits de luxe. Les élèves avaient pour consigne de

1. trouver, parmi des fiches horaires de train et d'avion présentées dans le dossier d'accompagnement de l'exercice, le transport qui correspondait aux attentes de l'employeur, listées dans une note de synthèse ;

2. rédiger un courriel à destination de leur supérieur afin de lui communiquer le résultat de ces recherches et de lui conseiller le meilleur transport possible ;

3. préparer ensuite un second courriel, de convocation, à destination des employés de l'entreprise, accompagné du programme du séminaire, ajouté au courriel en pièce jointe.

Les éléments à leur disposition pour la rédaction des deux courriels étaient :

- le scénario global fournissant le contexte (nom de la société, nom du gérant, motivation générale : organisation d'un séminaire) ;

- directives pour l'organisation du déplacement Lyon-Nice (arrivée impérative avant 12h);

- copies d'écran de recherches préalables pour un trajet en train et un trajet en avion, avec horaires et prix ;

- programme du séminaire fictif.

Les modalités de réalisation de ces deux messages électroniques étaient assez souples et la possibilité avait été donnée aux élèves de proposer des productions collectives. Certains messages ont donc été co-signés par trois ou quatre élèves. Certains élèves ont également choisi de constituer des groupes après avoir travaillé individuellement à un premier jet tandis que d'autres n'ont produit qu'une version. Certains élèves n'ont produit qu'un des deux écrits demandés, on ne trouve de ce fait pas toujours deux productions pour chaque élève, ce qui interdit une comparaison par élève des deux courriels.

Nous distinguerons dans notre analyse les deux courriels attendus : celui qui devait faire état des renseignements collectés sur l'hébergement et le transport sera désigné par "RéponseSupérieur »; celui qui convie les employés fictifs au séminaire, fictif lui aussi, sera désigné par «Convocation». Nous avons recueilli 29 productions: 17 pour RéponseSupérieur; 12 pour Convocation. Nous avons mobilisé pour l'analyse de ces messages des observables mettant en jeu aussi bien des attentes quant au genre que d'autres paramètres pragmatiques et linguistiques.

\subsection{Observables analysés}

Un des éléments de référence à prendre en compte est la prescription scolaire quant au genre du courriel. Qu'est-ce que la formation dispensée dans ce baccalauréat enseigne aux plans rhétorique, pragmatique et formel sur l'écriture du courriel ? Un manuel de Gestion- 
Administration propose une fiche ressource sur le message électronique qui en précise les caractéristiques, nous reproduisons une partie de ses indications (Fig. 1).

\section{Le style de rédaction et de présentation}

\section{Le message électronique professionnel doit respecter certaines règles. La netiquette définit les} règles de conduite et de politesse à adopter lors de la rédaction de messages électroniques.

- Il faut commencer par la formule d'appel. Il n'a pas besoin d'indiquer la date, le lieu, l'expéditeur et le destinataire comme sur un courrier classique. Cela se fait automatiquement; il suffit de remplir les fenêtres prévues à cet effet par la messagerie électronique. Le message sera automatiquement daté avec l'heure de son envoi.

La formule d'appel est plus directe surtout lorsque l'on a des relations professionnelles habituelles avec la personne. Par exemple, "Bonjour " ou le prénom de la personne est suffisant.

- L'objet du message est très précis.

- Le texte du message est concis et le style direct.

En cas de réponse à un courrier, reprendre quelques éléments du message original, ce qui facilitera la compréhension du correspondant.

- La formule de politesse est simplifiée (" Cordialement ", « Bien à vous ", " Sincères salutations ", "Courtoisement ", etc.);

- La signature : prénom et nom de l'expéditeur, éventuellement la fonction.

La rapidité de la rédaction ne doit pas faire oublier la relecture et la correction des fautes d'orthographe.

Fig. 1. Extrait d'un manuel pour les classes de Gestion-Administration

Nous retenons de cette fiche la prise en compte du genre qui se traduit par la présence, dans les courriels, d'une formule d'appel et d'une formule de politesse appropriées, d'un texte facilitant la compréhension du destinataire, notamment par un rappel des quelques éléments de contextualisation fournis par le dossier des élèves, d'une signature indiquant éventuellement la fonction.

On notera que, s'agissant d'un échange fictif, les élèves avaient à reconstruire entièrement à travers ce message une situation hypothétique, c'est-à-dire un destinataire imaginaire disposant plausiblement de certaines informations. Cette construction imaginaire d'un espace d'échanges n'est pas propre au courriel mais elle tient à la communication électronique. Marcoccia [12] note par exemple que, lors de l'écriture sur forum, la prise en compte d'un destinataire passe par l'élaboration mentale d'une « fiction de discussion ». Entre alors crucialement en jeu le respect des maximes conversationnelles de Grice [25] : le message se doit d'être suffisamment informatif, mais pas trop long, il doit être pertinent et globalement « coopératif».

Les quatre sortes d'observables que nous prenons en compte sont congruents avec les exigences de cette situation de communication.

1. Le respect du genre : Le message fait-il usage de formules d'appel et de politesse ? Comporte-t-il une signature ? Une indication sur le statut du scripteur?

2. L'implicite : Le message fournit-il des éléments de contextes pour son interprétation ? Qu'est-ce qui transparait dans le texte de l'état de connaissances supposé du destinataire ? Comment celui-ci est-il pris en compte ?

3. L'organisation du discours : Comment le texte est-il structuré, à la fois sur le plan typodispositionnel (présence de paragraphes) et sur le plan linguistique (connecteurs, organisateurs textuels) ? Comment organise-t-il les informations apportées ? 
4. Les choix énonciatifs de la relation au destinataire: Comment le scripteur se positionne-t-il ? Utilise-t-il « je », des tournures impersonnelles ? Comment s'adresset-il à son destinataire ? Comment marque-t-il la relation hiérarchique ?

Ces observables sont pour nous des indicateurs de la compétence communicationnelle des élèves et livrent des pistes pour les propositions didactiques que nous formulerons en fin d'article.

\section{Compétences et défaillances des élèves}

Avant de mettre en évidence les caractéristiques marquantes des textes des élèves, une analyse de la tâche cernera les choix possibles pour la réalisation des courriels et aidera à comprendre ce qui était en jeu dans la rédaction des deux messages.

Le scénario proposé impliquait pour chaque scripteur de déterminer le niveau de connaissances de son / ses interlocuteur-s. Le premier destinataire, l'employeur fictif, était supposé savoir de quoi il retournait, en tant que donneur d'ordre. Mais rien n'était dit dans le scénario sur le niveau d'information des destinataires du second message. Le scripteur devait décider des informations à inclure dans le message de "convocation ». Il devait aussi décider de la forme de ce message : un courriel collectif à l'ensemble des participants ou un courriel individuel à chaque participant?

Indépendamment du niveau d'information, il était nécessaire de fixer un degré de saillance supposé car même quand les informations sont connues, le destinataire n'a pas toujours l'ensemble des données présentes à l'esprit. C'est d'ailleurs pourquoi la fiche récapitulant les contraintes liées au mail (Fig. 1) stipule : « reprendre quelques éléments du message original, ce qui facilitera la compréhension du correspondant». Définir quoi reprendre et comment lier le connu de l'échange antérieur au nouveau du courriel à rédiger fait partie des problèmes d'écriture à résoudre.

De même, la situation du scripteur elle-même devait faire l'objet d'un choix : était-il inclus comme participant au séminaire prévu ou non? En fonction de ce choix, il pouvait être amené à s'inclure sur le plan énonciatif ou non, ce qui avait une incidence sur les pronoms utilisés.

\subsection{Des compétences communicationnelles manifestes}

\subsubsection{Respect du genre}

Pour la quasi-totalité des élèves, il est clair que les éléments indispensables du courriel comme outil de communication institutionnelle sont une évidence. Un seul message a omis la «formule d'appel» rituelle. Celle-ci est présente sous la forme «Bonjour» dans l'écrasante majorité des productions. Les scripteurs ont ainsi intégré le caractère moins formel du courriel qui autorise un style plus direct lorsque le message prend place dans un circuit d'échanges habituels (cf. Fig. 1). Rappelons que la situation fictive présentée aux élèves leur attribue un commanditaire masculin. Deux élèves ajoutent assez logiquement «Monsieur» à la formule d'appel : «Bonjour Monsieur » mais un autre utilise « Bonjour Madame ». Dans les deux cas les élèves ne se rendent pas compte que ce qui pourrait apparaitre comme une marque de politesse aboutit à produire un effet décalé, à la fois plus oral et plus protocolaire. Un autre élève commence son message par seulement « Madame » (peut-être ces «madame » s'adressent-ils à l'enseignante), qui tire alors le message vers les formes habituelles de la lettre.

La formule de politesse est elle aussi massivement présente, sous la forme "Cordialement», à l'exception d'un élève (différent de celui qui a omis la formule d'appel) qui n'écrit rien pour clore le message. Pour la signature, 23 messages adoptent la 
double mention du nom et de la fonction, comme il est recommandé, 5 adoptent le nom seul et 1 message ne comporte pas de signature.

On constate qu'au final, seuls trois messages sur les 29 recueillis ne sont pas totalement conformes aux attentes explicitées dans le manuel, en omettant l'un des éléments constitutifs du courriel. Ces jeunes scripteurs font ainsi la preuve de leur appropriation du genre sur le plan formel. Au-delà de cet aspect formel, des choix énonciatifs témoignent d'une certaine maitrise de la situation de communication.

\subsubsection{Situation de communication maitrisée}

Nous distinguerons ici les messages destinés à un supérieur hiérarchique et ceux destinés à des pairs (voir la présentation du corpus en 3.1), en ce qu'ils requièrent des positionnements énonciatifs différents, plus ou moins clairement programmés par l'exercice.

\section{Courriels fictivement adressés à un supérieur hiérarchique}

Ces courriels manifestent une réelle compréhension de la relation qui s'établit dans l'univers professionnel - alors, rappelons-le, que les scripteurs sont à peine lycéens et ne connaissent le monde de l'entreprise et ses codes que par les situations de stage. Dans 15 messages sur 17, on voit que l'auteur fonde les propositions qui constituent le cœur du message sur sa recherche d'informations. L'élève endosse ainsi un rôle d'assistant, qui consulte et analyse les informations disponibles et en dégage des conseils ou des préconisations. Le texte rend compte de ce traitement en l'articulant avec les propositions formulées, ce qui lui donne une teneur argumentative tout à fait pertinente dans la situation de communication (nous conservons dans chaque extrait la graphie produite par l'auteur) :

(1) J'ai prospécté les vols et les trains pour le trajet du séminaire, j'ai constaté que les vols étaient plus intéressants,

(2) Je me suis renseigné sur le mode de transport concernant le séminaire du 08/05 au 10/05. Le transport en avion me semblait plus intéressant autant sur le prix que sur la durée du voyage.

(3) J'ai regardé les vols et les train pour le seminaires à Nice. J'ai constaté que les vols étaient plus interresant. C'est pour cela que j'ai fait le choix de vous proposer le vol du lundi 8 mai à $8 \mathrm{~h} 30$

La formulation peut même adopter un registre assez soutenu, mais celle-ci contraste avec un choix lexical moins approprié. En (4), répondant à vos attentes indique habilement que les directives spécifiées par le scénario ont bien été prises en compte, mais je vous informe que est plutôt maladroit, s'agissant d'un acte de conseil ou de suggestion.

(4) Je vous informe que nous avons trouvé le moyen de transport répondant à vos attentes pour aller de Lyon à Nice.

Autre témoignage de la pleine compréhension de la communication en jeu, certains de ces courriels vont au-delà du scénario prescrit en ajoutant une offre de service, telle qu'on peut l'attendre d'un assistant ou secrétaire :

(5) Je suis à votre disposition si besoin pour réserver le vol ou de même pour refaire des recherches.

(6) pour une plus grande rapidité, je me propose donc de faire la réservation.

(7) Si vous acceptez ma proposition, je suis disponible à faire la réservation à votre place.

Mais là encore, on peut observer une discordance entre l'intention et la formulation :

(8) $0 \_-\_3-4 \_-10-77$ pour plus de renseignement veuiller me rappeler. 
L'intention en (8) est de marquer la disponibilité pour d'éventuels renseignements complémentaires, mais le pour plus de renseignements assez laconique et l'impératif veuillez produisent un effet de désinvolture sans doute non souhaité et non perçu.

\section{Courriels adressés à des pairs}

L'autre série de courriels, Convocation, révèle une plus grande difficulté pour les scripteurs à se positionner dans la communication. La consigne associée au courriel, "Préparer l'email de convocation des salariés sur le support ci-dessous », n'offrait pas un cadre aisé à trancher : le texte devait-il adopter le ton assez abrupt de la convocation ou celui plus avenant de l'invitation? Les jeunes scripteurs avaient le choix entre diverses postures. 11 sur 12 ont résolument opté pour le registre de l'invitation, plus conforme aux conventions de communication entre pairs :

(9) Nous vous invitons à participé au séminaire du 08/05/2017 au 10/05/2017 à Nice (grasse) pour l'usine de parfum.

(10) Nous souhaitons vous invitez au séminaire qui se déroulera à Grasse du 08/05/17 au $10 / 05 / 17$.

Un message toutefois s'embrouille dans une formule qui ne choisit pas, (11), tandis qu'un autre juxtapose invitation et convocation, (12), oscillations qui témoignent de la perplexité des élèves face à la tonalité énonciative à adopter :

(11) Je vous invite à être convoquer au Programme du Séminaire «Fragonard» du 08/05/2017 au 10/05/2017.

(12) Je vous écrit ce mail pour vous inviter au séminaire [...] Je souhaiterais vous convoquer $[\ldots]$ pour vous parler du deroulement de ce sejour.

Autre problème à résoudre, le message devait informer les participants du fait qu'ils seraient logés en chambres de 4 personnes et avaient donc à préciser leurs " colocataires" . $\mathrm{Si}$, pour certains, la gestion de cette information n'a pas posé de difficulté énonciative particulière, (13) à (15), d'autres ne l'ont pas traitée aisément, (16) et (17) :

(13) Je vous informe que les chambres sont pour 4 personnes, et je vous laisse m'informer avec qui vous souhaitez la partager.

(14) Les chambres sont pour 4 personnes, merci de nous préciser avec qui vous voulez être.

(15)Les chambres sont pour 4 personnes nous vous demandons donc votre avis pour savoir avec qui vous voulez être.

(16) Je vous informe que les chambres sont pour 4 personnes. Si vous le souhaitez on vous laisse choisir avec qui vous voulez être.

(17) Les chambres peuvent disposer de maximum 4 personnes. C'est pour cela que nous souhaîtons savoir rapidement avec qui vous souhaitez être?

Oubli ou évitement de la difficulté ? Trois messages ne font pas mention de cette question de chambres.

Ce second message à rédiger dans le cadre du scénario pédagogique mis en place laisse voir de réels contrastes entre les performances des différents élèves. Ces contrastes sont flagrants dans la structuration du message ou à travers les différents actes de langage.

\subsection{Des performances discursives contrastées}

Les deux courriels avaient une vocation d'information. Le scripteur devait donc organiser les informations fournies et gérer la demande de réponse. Les directives d'organisation du voyage incluses dans le dossier à traiter sous la forme d'une note de synthèse constituent un cadre structurant, en ce qu'elles offrent une trame chronologique : un trajet aller, un séjour, un trajet retour. Cette chronologie a largement servi de point d'appui pour la structuration 
des messages RéponseSupérieur qui massivement indiquent horaires et tarif pour le trajet Aller puis les mêmes données pour le trajet Retour (nous conservons le découpage en paragraphes original) :

(18) Le départ se déroulera le lundi à 8 h30 arrivé à 9 h52 pour $77 €$, et le retour le mercredi à 6 h55 arrivée à 7 h55 pour $49 €$.

(19) Le départ du vol est à 8 h30 le lundi et l'arivée est prévu pour 9h25. Le prix de l'aller est de $77 €$.

Le retour est le mercredi à 6 h55 et l'arrivée est prévue pour 7 h55. Le prix de votre vol est de $49 €$.

Certains élèves ont toutefois procédé à une réorganisation des informations collectées et rassemblent les données sur les horaires d'un côté, celles sur les prix de l'autre :

(20)Le départ est le lundi 8 mai et l'arrivée sera donc prévue pour 9 h25 à Nice, le trajet est court et permet donc votre arrivée avant $12 \mathrm{~h}$.

pour le vol du Retour, il est prévu pour $17 \mathrm{~h} 30$ le mercredi 10, l'arrivée est à $18 \mathrm{~h} 20$ à Lyon.

Le total par personne est de $165 €$ aller-retour, 15 personnes participants au séminaire, le coût est donc de $2475 €$.

(21) Je vous propose un vol avion.

Le depart sera Le Lundi à $15 \mathrm{~h} 15$ et l'arrive à $16 \mathrm{~h} 15$. (Lyon-Nice).

Le tragée vas durer $1 \mathrm{~h} 00$;

Le Retour sera le Mercredi à 06 :55 et l'arrivée à 7 h55 (Nice-Lyon).

Le prix allez est de $67 €$. Le prix retour est de $49 €$

On notera le contraste saisissant entre la capacité montrée là à se distancer de la chronologie pour procéder à une réorganisation des informations et les défaillances formelles des textes, nous y reviendrons en 4.3.

Deux messages toutefois omettent des informations essentielles par rapport au scénario, sur le retour et/ou sur le tarif. De ce fait, leur taille réduite pousse le scripteur à enchainer les propositions sans marquage explicite de lien logique ou chronologique, par ex. (22).

(22) Bonjour,

J'ai fait des recherches pour votre séminaire je vous propose un aller en train $\mathrm{n}^{\circ} 5103$ pour Nice. Votre voyage débutera à $9 \mathrm{~h} 05$ et se terminera à $10 \mathrm{~h} 31$, vous arriverez à temps pour déjeuner à $12 \mathrm{~h}$.

Cordialement

Un tel message manifeste un réel écart à ce qui serait attendu, à la fois en termes de quantité et de qualité, d'un échange coopératif [25]. C'est là une source d'hétérogénéité importante entre ces productions. Certaines apportent des éléments pertinents dans la situation fictive : une justification de la proposition, la proposition elle-même, les horaires des trajets, le prix unitaire et le prix total. D'autres sont tellement minimalistes, tout en comportant une ou des informations non pertinentes, comme le numéro du train en (22), qu'elles ne peuvent remplir l'objectif communicationnel assigné par le scénario. S'agit-il là d'un désinvestissement d'une tâche uniquement scolaire, sans réel enjeu de communication, ou d'une incapacité à estimer les informations pertinentes? Nous n'avons malheureusement pas pu interviewer ces élèves pour élucider la question.

Un dernier aspect qui contraste fortement les performances des élèves est la dimension rhétorique et énonciative. Nous avons déjà mentionné la bonne adéquation des textes à la situation de communication, en particulier en ce qui concerne la relation au destinataire (cf. 4.1.2) : certains messages font preuve de réelles habiletés, tandis que d'autres s'empêtrent quelque peu dans un positionnement incertain.

Cette hétérogénéité subsiste sur le plan rhétorique. Les textes les plus habiles s'ouvrent sur une mise en relief de la recherche effectuée, qui constitue alors le fondement 
argumentatif de la suite du message, ce que nous avons souligné en 4.1.2. Implicitement, le texte concourt alors à valoriser son auteur : son propos est étayé, fruit de la mise en œuvre de savoir-faire. À l'inverse, une première phrase de message telle que (23), qui ne s'appuie sur aucune remise en perspective ni justification, produit un effet de "hors de propos".

(23) Bonjour, Je vous conseilles de réserver le billet d'avion.

Se manifestent là des aptitudes différentes à gérer la dimension illocutoire. Certains élèves produisent des énoncés particulièrement appropriés, montrant une bonne appréhension des actes de langage en jeu, par exemple par l'emploi du passif en (24), ou par les modalisations de (25) et (26).

(24) Vous êtes invites au séminaire à Grasse du 8 mai 2017 au 10 mai 2017.

(25) Veuillez nous confirmez définitivement et le plus rapidement votre retour avant le 25 avril.

(26) $\mathrm{Si}$ vous accepter cette proposition je pourrais faire les réservation.

D'autres au contraire semblent naviguer dans un brouillard énonciatif, ne maintenant pas un positionnement assuré du début à la fin du message. Les courriels emploient en effet fort à propos le vouvoiement de rigueur pour le destinataire, qu'il s'agisse du supérieur hiérarchique ou de l'employé à " convoquer », mais hésitent parfois entre je et un nous dont on ne sait au final pas bien qui il désigne. Les extraits (27) et (28), dans lequel les deux nous successifs ne semblent pas avoir la même référence, en donnent des exemples.

(27) je vous informe que les chambres sont pour 4 et je vous laisse nous informer avec qui vous souhaitez être dans votre chambres.

(28) Je vous informe que nous avons trouvé le moyen de transport répondant à vos attentes pour aller de Lyon à Nice. Le vol n ${ }^{\circ}$ AF7839 d'aller sera le lundi 08 Mai 2017 à 0830 nous arriverons vers 09h25 à Nice le tarif est de 77€/pers.

Un seul message évite ces écueils en étant rédigé en totalité avec des tournures impersonnelles, montrant tout à la fois une réelle maitrise des tournures passives, sur les plans syntaxique comme énonciatif, et de vraies difficultés sur d'autres plans, choix lexicaux et gestion de l'implicite en particulier (voir 4.3). Nous le reproduisons en (29).

(29)Ce séminaire débutera le 08/05 et se terminera le 10/05/2017. 15 personnes y participeront. Le moyen de transport pour aller de Lyon à Nice sera fait en avion. Le vol de départ sera à $08 \mathrm{~h} 00$; pour le vol de retour, les personnes arriveront à $10 \mathrm{H}$. Le prix aller-retour sera de $90 €$.

Les divers extraits attestent de la coexistence, parfois dans un même texte, de réelles habiletés rédactionnelles et de défaillances de natures diverses. Ces dernières signalent des difficultés largement partagées.

\subsection{Néanmoins de réelles difficultés}

\subsubsection{Sur le plan formel}

Nous avons mentionné que Colin [10] a pu observer dans le discours d'enseignants de 3ème cycle primaire une tendance à se focaliser sur la «maitrise de la langue ». Si l'on s'intéresse aux performances des élèves de notre corpus dans les domaines de la syntaxe, de l'orthographe ou du vocabulaire, on peut effectivement relever des défaillances, comme l'illustrait (11), qui combine une tournure peu heureuse et une erreur orthographique :

Je vous invite à être convoquer au Programme du Séminaire «Fragonard»du 08/05/2017 au 10/05/2017.

En ce qui concerne l'orthographe, les extraits déjà mentionnés montrent que la plupart des élèves butent sur l'orthographe grammaticale, notamment les terminaisons des verbes 
conjugués au présent de l'indicatif, sur les marques d'accord dans le groupe nominal (voir par exemple (26), plus haut), sur les homophones grammaticaux tels que «à » et «a » ou sur les finales en $/ \mathrm{E} /$. Ces difficultés orthographiques, qui correspondent à des zones particulièrement problématiques de la langue française, sont spécifiquement discriminantes, comme le montrent Lucci et Millet [26], puisque les élèves les moins pourvus en capital scolaire y accordent moins d'attention que les autres.

D'autres erreurs tout aussi récurrentes, moins souvent explorées et liées aux aspects plus strictement textuels, apparaissent dans ces messages. Les élèves semblent rencontrer de réelles difficultés dans le domaine de la ponctuation, sur le plan stylistique comme sur le plan purement grammatical. La ponctuation à l'écrit peut exercer plusieurs fonctions et participe tant à la correction grammaticale d'un énoncé qu'à la construction de l'objet texte, au même titre que les connecteurs [27]. Les difficultés rencontrées par la plupart des élèves à ponctuer leurs textes relèvent ainsi au moins autant de la grammaire de texte que de la maitrise de la langue. Outre une gestion parfois erratique des virgules et points-virgules, par ex. (21), certaines productions contiennent des phrases sans point final :

(30) Je souhaite vous inviter au séminaire du 08/08/17 au 10/08/17

(31) Pouvez-vous me confirmer rapidement votre réponse, pour être définitivement sur de votre venue

À la lecture de ces productions écrites, on peut s'interroger sur le lien entre ces absences de ponctuation et la réelle difficulté qu'éprouvent ces élèves à structurer leurs textes. La notion de paragraphe comme unité sémantique et/ou grammaticale apparait comme particulièrement problématique à la lumière de certains messages qui comportent peu de paragraphes et semblent découpés de manière aléatoire. Certains élèves n'ont pas inséré de paragraphes du tout, le corps du texte se présente alors en un seul bloc, comme dans (32) :

(32) Bonjour,

J'ai chercher les vols et les trains pour le séminaire à Nice. Les offres les plus intéressantes étant les vols, mon choix s'est donc porté dessus, le départ est le lundi 8 mai à $08 \mathrm{~h} 30$ et l'arrivée est prévu pour $9 \mathrm{~h} 25$, le trajet n'est pas très long et permet donc votre arrivée avant $12 \mathrm{~h}$. Pour le vol du retour, il est prévu pour le mercredi 10 mai à $17 \mathrm{~h} 20$, l'arrivée est à $18 \mathrm{~h} 2 \mathrm{O}$. Le prix total par personne est de $165 €$ aller/retour.

A contrario, la plupart des élèves sont allés à la ligne, voire ont marqué des retraits, à chaque phrase, ce qui laisse une impression de paragraphes au sens typographique du terme, mais sans que l'on puisse nettement distinguer sur quoi repose l'unité dudit paragraphe. Il conviendrait néanmoins de vérifier si ces difficultés formelles se rencontrent également dans des genres écrits plus longs et plus construits que le courriel, sachant que leur manuel de gestion-administration lui-même n'offre comme exemples de couriels que des textes brefs et concis, construits en une ou deux phrases-paragraphes.

Au-delà des difficultés purement linguistiques qui se manifestent par le biais de maladresses lexicales et d'erreurs orthographiques, l'activité de mise en texte est donc une tâche problématique pour ces élèves de lycée professionnel. L'autre dimension qui les met en échec concerne la gestion de l'implicite.

\subsubsection{Pour la gestion des implicites}

Lahire [9], analysant des récits d'élèves en difficulté scolaire, émet l'hypothèse que la part importante laissée à l'implicite et l'emploi systématique de déictiques seraient le signe d'un rapport oral aux savoirs qui ne correspond pas aux attentes de l'institution scolaire, laquelle suscite et favorise un rapport qu'il appelle scriptural. À sa suite, nous nous sommes donc interrogées sur la capacité des élèves de lycée professionnel à décontextualiser puis recontextualiser les informations dans leurs productions écrites, ce qui revient à se 
conformer au manuel de gestion-administration, qui préconise de donner tous les éléments de contexte nécessaires à la compréhension du message (Fig. 1).

Il semble que cet aspect primordial de la compétence rédactionnelle a posé de réels problèmes à certains élèves qui n'ont pas su gérer, ou n'ont pas vu, la nécessité de recontextualiser leur message. La plupart d'entre eux font référence au séminaire dont il est question dans le scénario pédagogique par l'article défini le ou l'article contracté $a u$ : «le séminaire », " au séminaire ». Certains spécifient le nom par un complément précisant le lieu : "le séminaire à Nice », ou la date, parfois les deux : " le séminaire du 08/05 au 10/05 à Grasse », tandis que d'autres ne donnent aucun élément de contexte. Un élève ouvre directement le mail à son supérieur hiérarchique par le déterminant démonstratif $c e$, (29), plus haut, tandis qu'un autre emploie le possessif (33):

(33) J'ai fait des recherches pour votre séminaire je vous propose un aller en train(...).

Ces divers choix linguistiques laissent penser que les élèves considèrent que leur destinataire est capable d'inférer les différents implicites contextualisant cet échange. Pour les messages de RéponseSupérieur, nous supposons que les élèves considèrent que leurs productions, répondant à une note de synthèse sur papier qui figurait dans le dossier d'accompagnement, s'inscrivent donc dans un fil de discussion, et ne jugent pas vraiment nécessaire de recontextualiser. L'élève débutant par «ce séminaire » semble penser que le séminaire est hautement saillant dans la conscience du destinataire et que celui-ci n'a pas besoin d'être aiguillé dans la lecture.

En ce qui concerne les messages Convocation, il n'est nulle part précisé si les salariés ont eu à un moment ou à un autre connaissance de l'existence et donc de l'objet de ce séminaire. Les élèves ont eu à juger eux-mêmes du niveau d'information à disposition des destinataires et donc de l'implicite du message : un élève a complété séminaire par une relative adjectivale "au séminaire qui aura lieu à Grasse», un autre en a précisé l'intitulé : «du Séminaire 'Fragonard' du 08/05/2017 au 10/05/2017», mais aucun n'en précise l'objet. Nous faisons l'hypothèse soit que les élèves se sont construit une représentation erronée de la situation de communication, auquel cas c'est la simulation didactique qui leur a posé problème, soit qu'ils entretiennent aux savoirs et aux situations même scolaires un rapport oral-pratique auquel la sociodidactique gagnerait à s'intéresser.

\section{Conclusion}

Les contre-performances des élèves sur les aspects formels, à l'échelle phrastique comme à l'échelle du texte, ne répondent pas aux attentes de l'institution scolaire et peuvent donc faire écran à la saisie de leurs compétences. Pourtant, de nombreuses habiletés sont mises en œuvre par ces élèves, en particulier autour de la gestion de la communication dans sa dimension pragmatique. La plupart des élèves semblent avoir intériorisé un certain nombre de routines langagières leur permettant d'effectuer efficacement les actes de langage visés par la tâche et de nombreuses productions manifestent une réelle habileté rhétorique, dans la justification des choix de transports par exemple.

Ces compétences, qu'à la suite de [11-13], nous qualifiions d'ignorées, gagneraient sans doute à être valorisées, d'abord parce qu'elles participent d'une réelle compétence professionnelle : l'efficacité dans la communication, ensuite parce que leur valorisation pourrait améliorer le rapport à l'écrit de ces mêmes élèves, en réduisant un potentiel sentiment d'insécurité scripturale. Ces compétences ignorées et inégalement partagées par les élèves pourraient donc constituer un levier didactique en ce qu'elles renforceraient la motivation des élèves et pourraient, en étant retravaillées en classe, être consolidées et transférées dans d'autres domaines. Nous envisageons donc la poursuite du travail sur deux versants.

Sur le plan didactique, le caractère fictif de la situation simulée semble avoir été un obstacle pour certains élèves. Le brouillage énonciatif dans certains mails de convocation 
ainsi que l'emploi de «Bonjour Madame», qui semblait s'adresser à l'enseignante, laissent en effet supposer que certains élèves ne sont pas parvenus à se projeter dans cette simulation didactique. Des situations représentant un véritable enjeu de communication, avec un destinataire effectif, sont indéniablement préférables. C'est pourquoi, dans l'esprit de valorisation des compétences acquises par les pratiques extra-scolaires et afin de leur permettre de développer de nouvelles compétences à travers un enjeu motivant, une expérimentation didactique est envisagée autour de l'écriture et de la publication sur les réseaux sociaux d'une chronique. Ce genre, très prisé de ces élèves, permet l'inscription dans un projet d'écriture longue, toutefois découpée en épisodes, et leur offrira la possibilité de construire des compétences transverses, en s'adressant à de réels destinataires. Le dispositif prévu articulera des phases d'écriture en atelier, combinant des écrits de différents genres, aussi bien fictionnels que professionnels, et des séances de travail sur les points précis de langue qui sont pour eux les plus problématiques. Ces points de langue, choisis en fonction des erreurs observées dans les productions d'élèves, seront abordés dans une perspective de remédiation et le travail devrait permettre aux élèves de développer des stratégies de correction plus efficaces. Nous faisons le pari qu'un atelier d'écriture intégrant des pratiques réflexives autour de la production écrite facilitera pour ces élèves un changement de posture et l'entrée dans un rapport écrit au savoir [22]. En effet, des dispositifs tels que la révision entre pairs et un étayage par des activités de réécriture amèneront les élèves à adopter, au fil des séances, des postures variées : de l'auteur à l'éditeur en passant par le lecteur. Ces variations de posture visent autant à susciter un rapport plus réflexif à leur pratique d'écriture qu'à leur donner plus d'assurance en tant que sujet-scripteur. L'expérimentation doit être menée pendant le premier semestre de 2018 et fait partie du travail doctoral de Cindy de Amaral.

Parallèlement, l'analyse de productions de genres diversifiés de ces mêmes élèves permettra de poursuivre l'exploration des spécificités de leur écriture et de mettre en évidence les zones les plus résistantes de la langue pour ces élèves à faible capital scolaire. Nous avons vu à travers ces courriels qu'au delà de la question orthographique, la mise en texte, la gestion de l'implicite sont particulièrement problématiques. Il reste à déterminer si ces difficultés subsistent dans des écrits d'autres natures, impliquant des contextes de communication différents. L'expérimentation projetée nous fournira ainsi des données appropriées pour continuer ces recherches, en produisant des textes fictionnels ou semifictionnels à teneur narrative à côté des écrits professionnels dont la maitrise est nécessaire pour leur scolarité.

\section{Références bibliographiques}

1. Cros, F. (2009). L'écriture, entre développement professionnel et développement personnel. Vie sociale, 2(2), 23-34.

2. Lafont-Terranova, J. (2009). Se construire, à l'école, comme sujet-écrivant: l'apport des ateliers d'écriture. Namur: Presses universitaires de Namur.

3. Barré-de Miniac, C. (2008). Le rapport à l'écriture: une notion à valeur heuristique. In S. Chartrand, C. Blaser, éd., Le rapport à l'écrit : un outil pour enseigner de l'école à l'université Namur: Presses universitaires de Namur, 11-23.

4. Delgery, A. (1999). Les représentations des difficultés en écriture. Spirale, 23, 111-123.

5. Palheta, U. (2011). Enseignement professionnel et classes populaires : comment s'orientent les élèves « orientés ». Revue Française de Pédagogie, 175, 59-72.

6. Cnesco (2016). De vraies solutions pour l'enseignement professionnel. Dossier de synthèse. https://www.cnesco.fr/fr/dossier-enseignement-professionnel/ [consulté le 31/03/2018]

7. Jellab, A. (2008). Sociologie du lycée professionnel : l'expérience des élèves et des enseignants dans une institution en mutation. Toulouse: Presses Universitaires du Mirail. 
8. Charlot, B. (1999). Le rapport au savoir en milieu populaire: une recherche dans les lycées professionnels de banlieue. Paris: Anthropos.

9. Lahire, B. (1993). Culture écrite et inégalités scolaires: sociologie de l'"échec scolaire" à l'école primaire. Lyon: Presses Universitaires de Lyon.

10. Colin, D. (2014). Analyse des pratiques d'écriture dans le discours des enseignants : enjeux didactiques: analyse de déclarations d'enseignants de CM2 et de 6ème, Doctorat Nouveau Régime, Université d'Orléans.

11. Elalouf, M.-L. (2012). Les connaissances cachées développées par la lecture et l'écriture électronique extrascolaires: enjeux pour la didactique du français. Études de linguistique appliquée, 166(2), 139-141.

12. Marcoccia, M. (2012). Définitions et négociations de la norme scripturale dans un forum de discussion d'adolescents. Études de linguistique appliquée, 166(2), 157-169.

13. Penloup, M.-C. (2007). Les connaissances ignorées: approche pluridisciplinaire de ce que savent les élèves. Lyon: Institut National de Recherche Pédagogique.

14. Brahic, M. (2009). Mieux rédiger ses écrits professionnels: lettres, messages électroniques, comptes rendus, rapports, analyses et synthèses. Paris: Eyrolles.

15. Bouquet, B., Riffault, J., éd. (2009). Les écrits professionnels. Pratique des écrits, écritures des pratiques. Vie sociale, 2(2), 164.

16. Beaudet, C., Leblay, C., Rey, V. (2016). L'écriture professionnelle. Pratiques, 171-172.

17. Laborde-Milaa, I., Plane, S., Rinck, F., Sitri, F., éd. (2014). La formation aux écrits professionnels Des écrits en situation de travail aux dispositifs de formation. Le discours et la langue, 5(2).

18. Rinck, F., Sitri, F. (2012). Pour une formation linguistique aux écrits professionnels. Pratiques, 153-154, 71-83.

19. Labasse, B. (2009). L'écrit professionnel: ambiguités et identités d'un objet académique. Pratiques, 143-144, 233-248.

20. Boch, F., Sorba, J., Bessonneau, P. (2016). Évaluer les compétences rédactionnelles : que tester? Le français aujourd 'hui, 193(2), 127-144.

21. Blanc, M., Dezalay, T., Ayard, C. (2002). Pratiques professionnelles et usages des écrits électroniques. Paris: L'Harmattan.

22. Lahire, B. (2008). La raison scolaire: école et pratiques d'écriture, entre savoir et pouvoir. Rennes: Presses Universitaires de Rennes.

23. Gajewska, E. (2016). Préparer à communiquer par écrit dans l'entreprise : courrier traditionnel vs courrier électronique. Pratiques, 171-172.

24. Labbé, H., Marcoccia, M. (2005). Communication numérique et continuité des genres: l'exemple du courrier électronique. Texto! Textes et Cultures, 10(3).

25. Grice, H. P. (1979). Logique et conversation. Communications, 30, 57-72.

26. Lucci, V., Millet, A. (1994). L'orthographe de tous les jours. Paris: Honoré Champion.

27. Adam, J.-M., Revaz, F. (1989). Aspects de la structuration du texte descriptif: les marqueurs d'énumération et de reformulation. Langue Française, 81, 59-98. 